\title{
GNSS Array Antenna for Mitigating Multipath Errors in Urban Environment
}

\author{
Toshiaki Tsujiii, ${ }^{1, *}$, Kenta Yonebayashi ${ }^{2}$, Takeshi Fujiwara ${ }^{3}$, and Sohshi Ohsawa ${ }^{3}$ \\ ${ }^{1}$ Department of Aerospace Engineering, Osaka Prefecture University, Sakai City, Osaka, Japan \\ ${ }^{2}$ Department of Aerospace Engineering, Tokyo Metropolitan University, Hino City, Tokyo, Japan \\ ${ }^{3}$ Aeronautical Technology Directorate, Japan Aerospace Exploration Agency, Mitaka City, Tokyo, Japan
}

\begin{abstract}
GNSS signal vulnerability has been a major concern especially for safety-of-life applications such as aircraft operations. Therefore, a GNSS array antenna technology was investigated focusing on beamforming to mitigate multipath errors in urban environment including airport surfaces. A commercial three-element GNSS antenna and RF-Front end were used to obtain digital IF data. The recorded Inphase/Quadrature IF data for three antennas were combined with proper weight to form beams towards satellites after so-called hardware biases were calibrated. Test results in multipath-rich environment demonstrated that $50-70 \%$ reduction of pseudorange errors due to multipath were possible if the beamforming algorithms were applied.
\end{abstract}

\section{Introduction}

Although GNSS has been widely used for navigation and positioning of various vehicles, its vulnerabilities to multipath, ionospheric anomalies, intentional and/or unintentional radio interference, etc. are big challenges especially for safety-of-life applications such as aircraft operations. Therefore, a GNSS array antenna technology was investigated focusing on beamforming to mitigate multipath errors in urban environment including airport surfaces. In this paper, a methodology to form beams to aimed satellites is introduced first. Then our test equipment including a commercial three-element GNSS antenna and a multi-input RF-Front end are shown. One of most important challenges for beamforming technique is to calibrate biases between antennas as well as frontends. The bias calibration method and test results are shown next. Finally, multipath mitigation performance using antenna array with hardware biases calibrated will be demonstrated.

\section{Array Antenna}

In this section, the concept of array antenna is briefly introduced. A linear N-elements array is considered as shown in Fig. 1. When a satellite is observed in a direction of azimuth $(\varphi)$ and zenith angle $(\theta)$, the received signal at the reference point is assumed as $E_{0}(t)$. Then received signal by the i-th element can be expressed as

$E_{i}(t)=E_{0}\left(t-\tau_{i}\right) \quad(i=1,2, \ldots, N)$

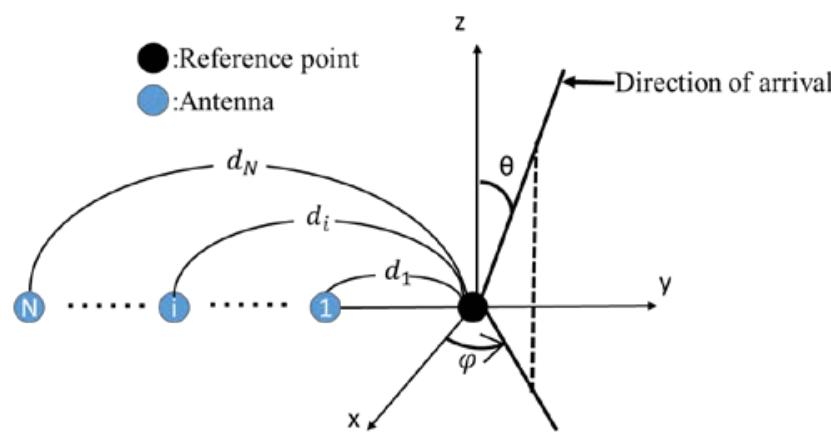

Fig. 1. N-element linear antenna array

In Eq. 1, $\tau_{i}$ is propagation delay with respect to the reference point, and given by

$$
\tau_{i}=\frac{d_{i} \sin \theta \sin \varphi}{c}
$$

where $d_{i}$ is distance of $\mathrm{i}$-th element from the reference point, and $c$ is speed of signal propagation. Therefore, Eq. (1) is expressed as

$$
\begin{aligned}
E_{i}(t) & =E_{0}\left(t-\tau_{i}\right) \\
& =E_{0}(t) \exp \left(-j 2 \pi f \tau_{i}\right) \\
& =E_{0}(t) \exp \left(-j 2 \pi f \frac{d_{i} \sin \theta \sin \varphi}{c}\right)
\end{aligned}
$$




$$
=E_{0}(t) \exp \left(-j 2 \pi \frac{d_{i} \sin \theta \sin \varphi}{\lambda}\right)
$$

where $f$ and $\lambda$ are frequency and wavelenght of the signal, respectively. The purpose of array antenna in this research is to strengthen the signal of aimed satellite by adjusting phase delay at each antenna. The signal at i-th antenna $\left(E_{i}(t, \theta, \varphi)=E_{i}(t)\right)$ is delayed by $\tau_{i}$ with respect to the signal at the reference point. To compensate this delay, the phase advance factor is multiplied as follows :

$$
\begin{gathered}
E_{i}(t, \theta, \varphi) \exp \left(\mathrm{j} 2 \pi \frac{d_{i} \sin \theta \sin \varphi}{\lambda}\right), \\
(i=1,2, \cdots, \mathrm{N}) \#(4) .
\end{gathered}
$$

The adjusted signals of $\mathrm{N}$ elements are summed and combined signal can be expressed as

$$
\begin{aligned}
& E_{\text {sum }}(t, \theta, \varphi) \\
= & \sum_{i=1}^{N} E_{i}(t, \theta, \varphi) \exp \left(\mathrm{j} 2 \pi \frac{d_{i} \sin \theta \sin \varphi}{\lambda}\right) \# \\
= & N E_{0}(t, \theta, \varphi)
\end{aligned}
$$

The signal phases from $(\theta, \varphi)$ direction are adjusted to allign, and as a result the combined signal is amplified by $\mathrm{N}$ times.

An example gain pattern of a 6-element linear array antenna with half wavelength separation is shown in Fig. 2. Multipath error would be mitigated by directing the main lobe towards the aimed satellite.

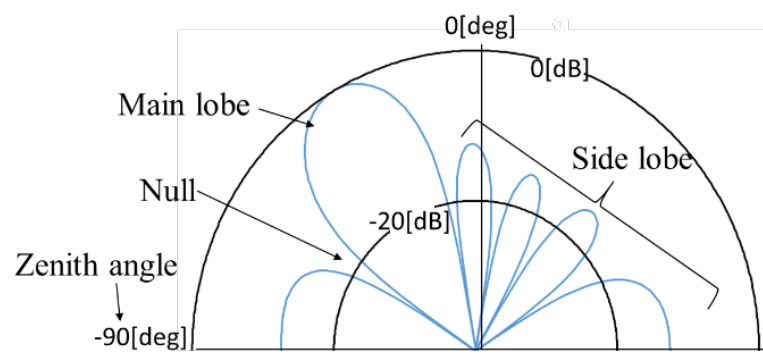

Fig. 2. Example gain pattern of a 6-elemnet linear array antenna

\section{Beamforming Algorithms}

In this research, a three-element antenna array was used and each antenna was connected to three RF front ends where a reference clock was commonly used. Intermediate frequency (IF) data for three antennas were collected by a PC as shown in Fig. 3. IF data consists of In-phase and Quadrature data, and they are combined to form a beam by using the following method. Fig. 4 illustrates the geometry of the array antenna and signal direction. Antenna 1 was defined as reference antenna, and the signal phases for other two antennas were adjusted to align to the reference antenna.

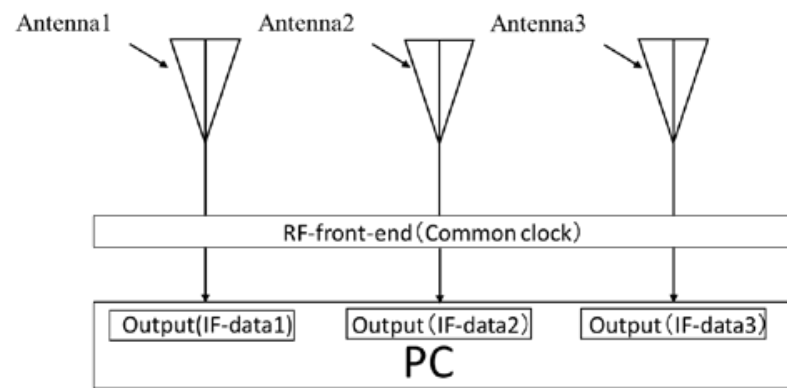

Fig. 3. IF data recording system for a three-element array antenna

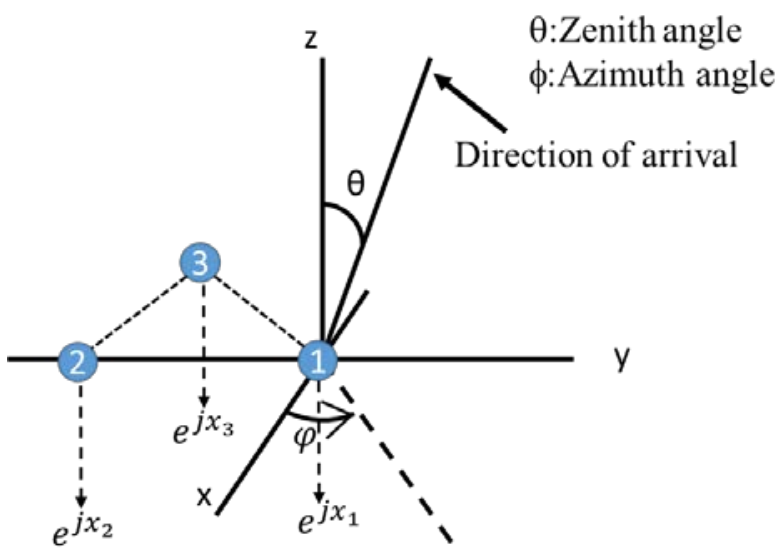

Fig.4. Geometry of 3-element antenna and signal direction

The signal from i-th antenna element can be expressed by the following equation.

$$
e^{j x_{i}}=I_{i}+j Q_{i}
$$

Now, phase differences between 2nd/3rd antennas and the reference antenna defined as $\delta_{21}, \delta_{31}$. Then the signal of second antenna is adjusted for its phase to align to the reference signal as follows:

$$
\begin{aligned}
e^{j x_{2}} \times & e^{+j \delta_{21}} \\
= & \left(I_{2}+j Q_{2}\right)\left(\cos \delta_{21}+j \sin \delta_{21}\right) \\
= & \left(I_{2} \cos \delta_{21}-Q_{2} \sin \delta_{21}\right) \\
& +j\left(Q_{2} \cos \delta_{21}+I_{2} \sin \delta_{21}\right)
\end{aligned}
$$

Then the signal of third antenna is also adjusted as follows:

$$
\begin{aligned}
e^{j x_{3}} \times & e^{+j \delta_{31}} \\
= & \left(I_{3}+j 3\right)\left(\cos \delta_{31}+j \sin \delta_{31}\right) \\
= & \left(I_{3} \cos \delta_{31}-Q_{3} \sin \delta_{31}\right) \\
& +j\left(Q_{3} \cos \delta_{31}+I_{3} \sin \delta_{31}\right)
\end{aligned}
$$

Finally, three I/Q data are summed to obtain new I/Q data for a beam-formed signal according to the following equations. 


$$
\begin{aligned}
& I^{\prime}=I_{1} \\
& \quad+\left(I_{2} \cos \delta_{21}-Q_{2} \sin \delta_{21}\right) \\
& \quad+\left(I_{3} \cos \delta_{31}-Q_{3} \sin \delta_{31}\right) \\
& \quad \\
& Q^{\prime}=Q_{1} \\
& \quad+\left(Q_{2} \cos \delta_{21}+I_{2} \sin \delta_{21}\right) \\
& \quad+\left(Q_{3} \cos \delta_{31}+I_{3} \sin \delta_{31}\right)
\end{aligned}
$$

\section{Experiments and Results}

\subsection{Hardware bias calibration}

In order to form proper directional beam, hardware biases due to antenna, antenna cable, RF front end have to be calibrated. In this research, the hardware biases were estimated by comparing carrier phases measured at antenna elements. Fig. 5 shows the three-element array antenna used for this experiment.

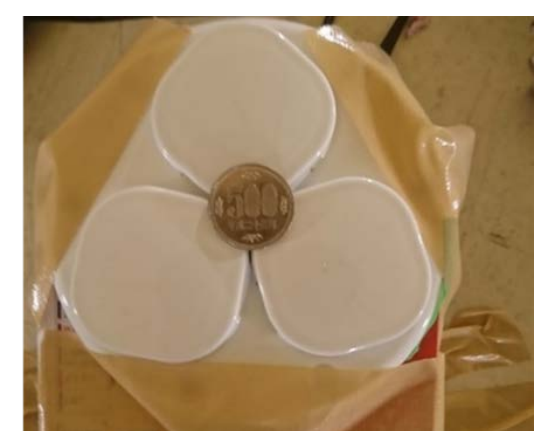

Fig. 5. Three-element array antenna

When carrier phase measurements for the first and i-th antenna are expressed by $\varphi_{1}, \varphi_{i}$, the hardware bias of the i-th antenna with respect to the first antenna $\left(b_{i 1}\right)$ can be calculated as follows

$$
b_{i 1}=\varphi_{i}-\varphi_{1}-L_{i 1} \quad(\mathrm{i}=2,3)
$$

where $L_{i 1}$ is difference of geometrical distances between the satellite and i-th/first antennas calculated theoretically.

The hardware biases of antenna-2/3 relative to antenna- 1 calculated by using carrier phase measurements of four satellites (G11, G17, G22 and G23) are shown in Fig. 6 and Fig. 7. The average of these biases are used for calibration.

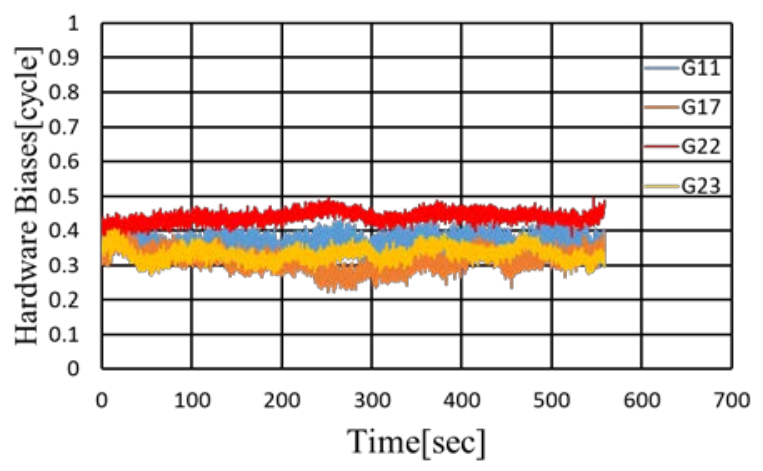

Fig. 6. Hardware bias of antenna-2 relative to antenna-1

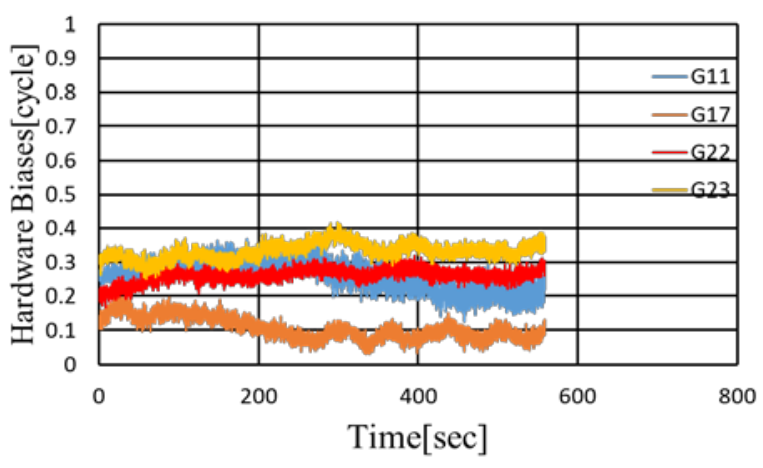

Fig. 7. Hardware bias of antenna-3 relative to antenna-1

In order to verify the efficacy of hardware bias calibration, theoretical calculation of received signal was compared with observation result. First, the expected signal strength variation before calibration was calculated (Fig. 8). The values at the right of color bar from -10 to $+10(\mathrm{~dB})$ indicate increment/decrement from the signal strength received by single antenna. Although phase adjustment for beamforming was not conducted, a gain pattern was seen. This pattern seemed to be caused by uncalibrated hardware biases. On the other hand, Fig. 9 shows the observed signal strength variation where the values at the right of color bar indicate $C / \mathrm{N}_{0}$ variation by a software receiver used for processing the IF data. The signals of satellites in eastern sky were weakened and strong correlation in signal strength variation can be seen between Fig. 8 and Fig. 9.

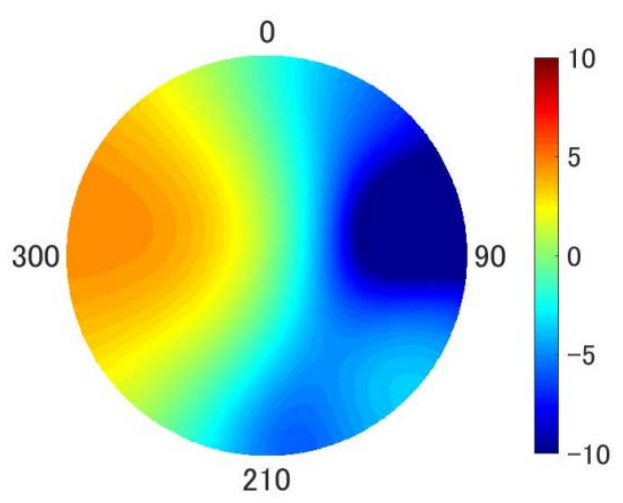

Fig. 8. Expected signal strength variation (biases NOT calibrated)

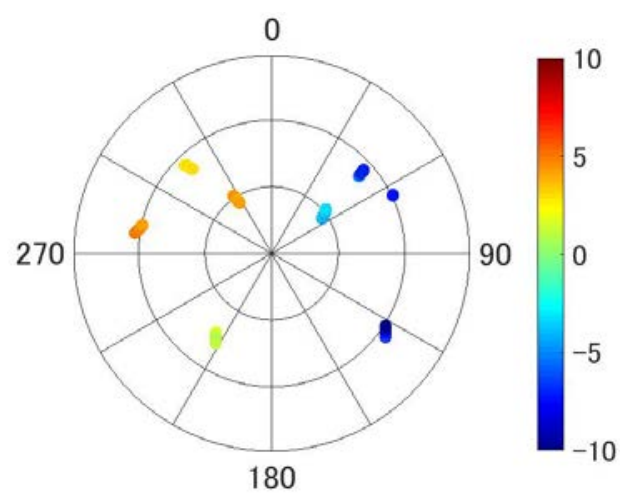

Fig. 9. Observed signal strength variation (biases NOT calibrated) 
Next, the expected signal strength variation after calibration was calculated (Fig. 10). Since no beamforming adjustment was carried out, the signal from the zenith was strongest and unsymmetrical gain pattern was not seen as expected. Fig. 11 shows the observed signal strength variation after bias calibration. Compared to the results shown in Fig. 9, the signals of satellites in eastern sky were enhanced in this case and showed similar tendency to Fig. 10. These results show the efficacy of bias calibration and now it is ready to proceed to beam forming.

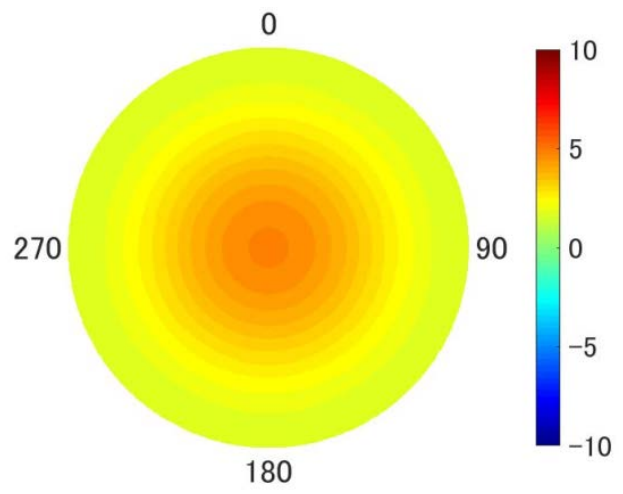

Fig. 10. Expected signal strength variation (biases calibrated)

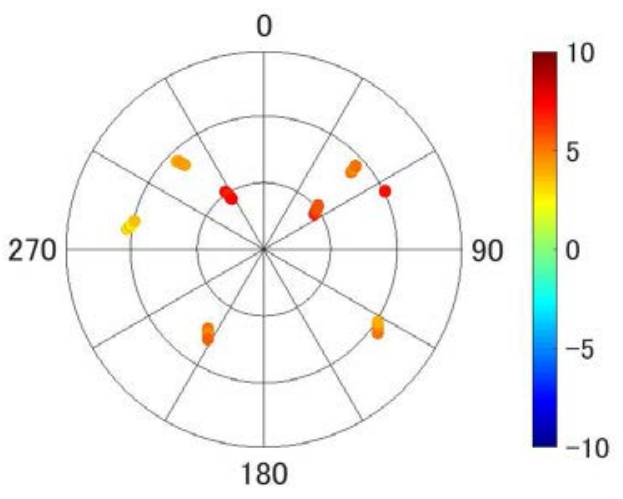

Fig. 11. Observed signal strength variation (biases calibrated)

\subsection{Multipath mitigation testing}

Before conducting multipath mitigation testing, GPS data were collected in open sky environment and hardware biases were estimated. Then test equipments were moved to multipath-rich environment. The satellite locations in the sky are shown in Fig. 12 while the map of test area is shown in Fig. 13. The GPS data were collected nearby four-story building and a large hunger for aircrafts.

Multipath effects were evaluated by taking difference between carrier phase and pseudorange (CMC, Codeminus-Carrier). Since multipath error on carrier phase is less than $1 / 4$ of wavelength, CMC indicates the multipath error on pseudorange which reaches to tens of meters.

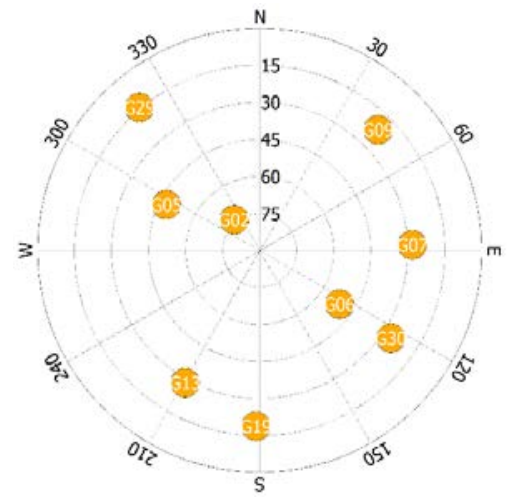

Fig. 12. Sky plot of GPS satellites

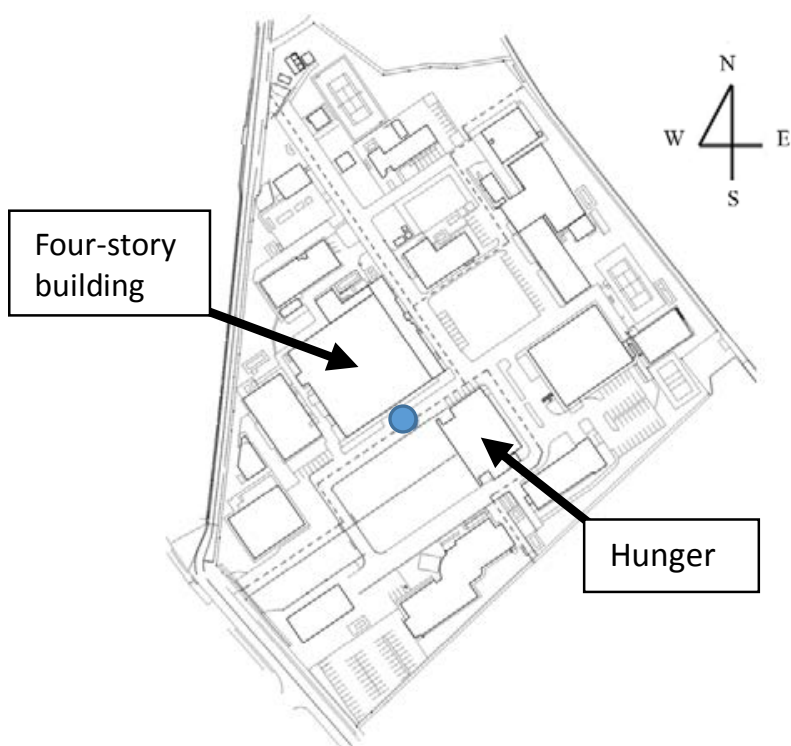

Fig. 13. Map of test area ( $\bigcirc$ : Data logging point)

The variation of CMC for GPS02 satellite is shown in Fig. 14. Since carrier phase has ambiguity, only CMC variation from the initial time is shown. The CMC for three-element array antenna is indicated by orange line, while the CMC calculated by using a geodetic receiver with single antenna is shown by blue line. Multipath mitigation by the beam-formed array antenna can be seen. The CMC variations for other satellites (GPS06 and GPS13) are also shown in Fig. 15 and 16, respectively. The effect of beam forming can be seen for these satellites, too.

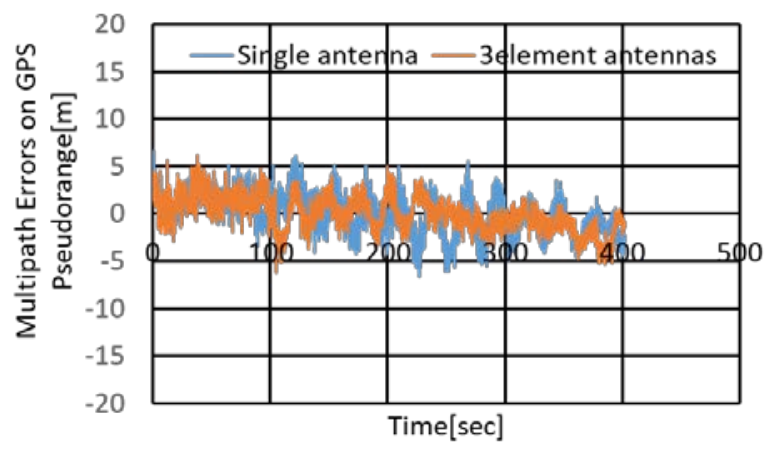

Fig. 14. Multipath error variation (G02) 


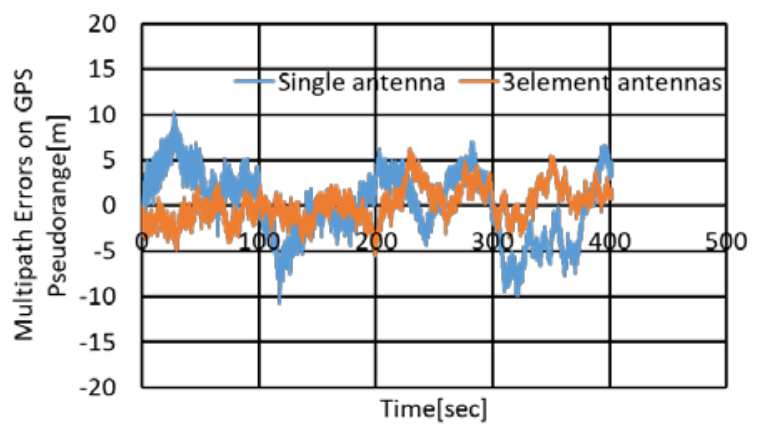

Fig. 15. Multipath error variation (G06)

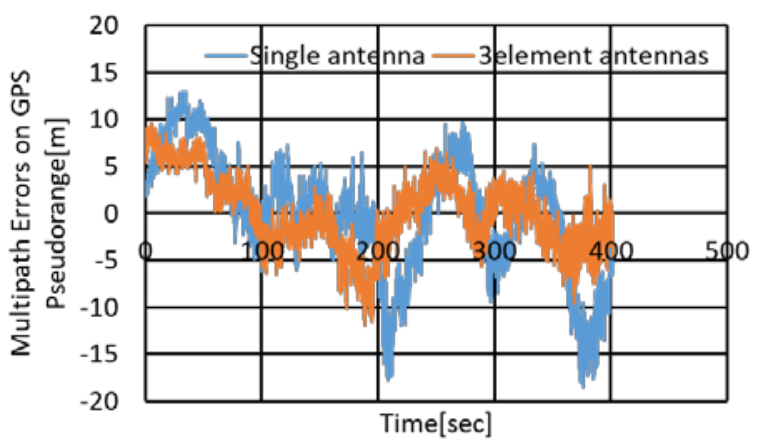

Fig. 16. Multipath error variation (G13)

Table 1 summarized the standard deviation (s.t.d) of CMC in this experiment. The first raw shows the s.t.d for an element of the array antenna (uncombed signal) while the second raw shows the s.t.d for the single geodetic antenna. The multipath error variations for two kinds of single antenna were similar. On the other hand, the CMC variation for the beam formed signal (in the third raw) showed smaller s.t.d, and therefore, a superior performance of array antenna for multipath mitigation was demonstrated.

Table 1 Standard deviation of pseudorange multipath error

variation
\begin{tabular}{|l|c|c|c|}
\hline standard deviation $[\mathrm{m}]$ & $\mathrm{G} 02$ & $\mathrm{G} 06$ & $\mathrm{G} 13$ \\
\hline 3element antennas (non-synthetic) & 3.41 & 4.66 & 6.77 \\
\hline Single antenna & 2.44 & 4.50 & 6.04 \\
\hline 3element antennas (synthetic) & 1.88 & 1.91 & 3.95 \\
\hline
\end{tabular}

\section{Conclusion and Future Work}

In this paper, the hardware bias calibration method by using carrier phase was validated. Then multipath mitigation experiment was conducted in the area surrounded by buildings. Test results demonstrated that $50-70 \%$ reduction of pseudorange errors due to multipath were able to be achieved if the beamforming algorithms were applied.

In the future work, the same algorithms will be applied to a seven-element array antenna (Fig. 17).

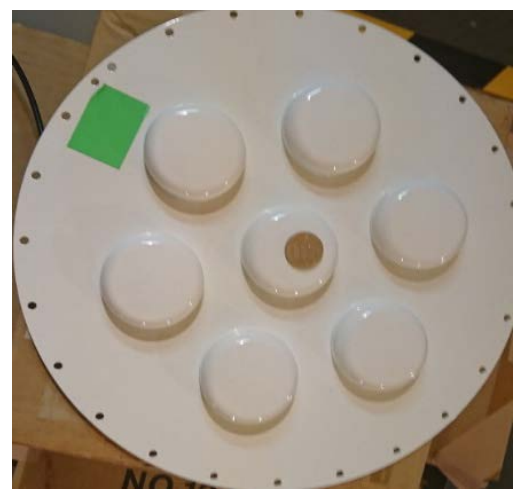

Fig. 17. Seven-element array antenna

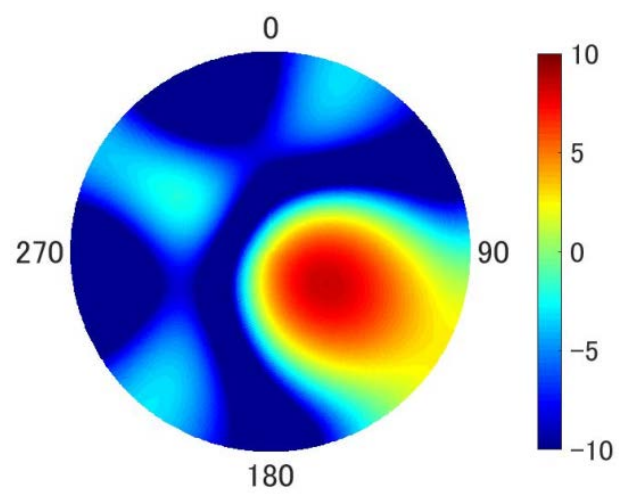

Fig. 18. Expected signal strength variation for sevenelement array antenna

Due to the effect of many elements and smaller separation $(9.5 \mathrm{~cm})$ between elements, forming a sharper beam is possible (Fig. 18) and further multipath mitigation can be expected.

This work was supported by JSPS KAKENHI Grant Number JP16K06550.

\section{References}

1. Kikuma, Nobuyoshi., Adaptive Signal Processing by Array Antenna, Kagaku Gijutsu Shuppan, 2004.

2. Ung Suok Kim, Dennis Akos, Per Enge, Frederic Bastide, "Simulation and Validation of a GPS Antenna Array Concept for JPALS Application", ION GPS/GNSS 2003, 9-12 September 2003, Portland,OR p1852-1855.

3. Saeed Daneshmand, Negin Sokhandan, Mohammad Zaeri-Amirani and Gérard Lachapelle, ” Precise Calibration of a GNSS Antenna Array for Adaptive Beamforming Applications”, Sensors 2014, 14(6), 9669-9691 